\title{
Processing overlap-dependent distractor dilution rather than perceptual target load determines attentional selectivity
}

\author{
Jinfeng $\operatorname{Tan}^{1,2} \cdot$ Shouhang Yin ${ }^{1} \cdot$ Lijun Wang $^{1} \cdot$ Antao Chen $^{1} \cdot$ Tobias Egner $^{3}$
}

Published online: 2 July 2018

(C) The Psychonomic Society, Inc. 2018

\begin{abstract}
The perceptual load theory of attentional selection argues that the degree to which distractors interfere with target processing is determined by the "perceptual load" (or discrimination difficulty) of target processing: when perceptual load is low, distractors interfere to a greater extent than when it is high. A well-known exception is load-independent interference effects from face distractors during processing of name targets. This finding was reconciled with load theory by proposing distinct processing resources for faces versus names. In the present study, we revisit this effect to test (a) whether increasing the processing overlap (perceptual, lexical, conceptual) between potential targets and distractors would reinstate the classic load effect, and (b) whether this data pattern could be better explained by load theory or by a rival account that argues that distractor dilution rather than target load determines the degree of distractor interference. Over four experiments, we first replicate the original finding and then show that load effects grow with increasing processing overlap between potential targets and distractors. However, by adding dilution conditions, we also show that these processing overlap dependent modulations of distractor interference can be explained by the distractor dilution perspective but not by perceptual load theory. Thus, our findings support a processing overlap dilution account of attentional selection.
\end{abstract}

Keywords Selective attention $\cdot$ Distraction $\cdot$ Dilution $\cdot$ Perceptual load

Jinfeng Tan and Shouhang Yin contributed equally to this work.

Significance Statement: The ability to selectively attend to task-relevant stimuli (targets) and ignore task-irrelevant (distractor) information is crucial for successfully navigating everyday life. The circumstances that determine the efficiency of this selection process are not entirely understood, however. The current study shows that the degree to which people successfully ignore distracting information depends both on the number of potentially relevant and irrelevant stimuli encountered as well as on their similarity.

Antao Chen

xscat@ swu.edu.cn

1 Key laboratory of Cognition and Personality of the Ministry of Education, Faculty of Psychology, Chongqing Collaborative Innovation Center for Brain Science, Southwest University, Beibei District, Chongqing 400715, China

2 College of Education Science, Hubei Normal University, Huangshi, Hubei 435000, China

3 Center for Cognitive Neuroscience, Department of Psychology and Neuroscience, Duke University, Durham, NC, USA

\section{Introduction}

Selective attention, the ability to focus on task-relevant (target) and ignore task-irrelevant (distractor) information, is critical for navigating everyday life. A central topic of debate in the field of selective attention has been the locus of attentional filtering within the stream of information processing. On one hand, early-selection accounts suggest that a distractor is filtered or attenuated at an early, perceptual level of analysis (Broadbent, 1958; Treisman, 1960). On the other hand, according to late-selection accounts, distractors are processed to the same degree as target information up to a post-perceptual processing stage, where memory and response selection processes become effective (Deutsch \& Deutsch, 1963).

Much work over the past two decades, however, suggests that the locus of attentional selection is in fact flexible, in that it can occur either early or late, as a function of the specific task requirements. Accordingly, the "perceptual load theory" of attention (Lavie \& Tsal, 1994; Lavie, 1995) proposes that selectivity arises when task-relevant processing consumes all 
available perceptual-attentional resources, as otherwise those resources will "spill over" to the processing of task-irrelevant features. Therefore, in situations where task-relevant processing is of "high load" (e.g., it is difficult to perceive the target stimulus), attentional capacity is fully engaged in processing task-relevant stimuli, and thus no spare resources are available for processing task-irrelevant stimuli, leading to early selection effects. By contrast, in situations of low perceptual load (i.e., an easily distinguishable target stimulus), the processing of task-relevant stimuli consumes only minimal attentional resources, such that the remaining resources "spill over" to process task-irrelevant distractors, leading to late selection effects (Lavie, 2005, 2010).

This theory has typically been tested by measuring the effects of varying perceptual target processing load on interference effects from incongruent (as compared to congruent or neutral) distractor stimuli. For instance, participants may be required to indicate, per button press, whether a centrally displayed target letter is an $\mathrm{X}$ or a $\mathrm{Z}$, while ignoring a vertically off-set distractor letter (also $X$ or $Z$ ) that can be either congruent (e.g., a distractor $\mathrm{Z}$ accompanying a target $\mathrm{Z}$ ) or incongruent with the target letter (e.g., a distractor $X$ accompanying a target $\mathrm{Z}$ ). The putative perceptual load of target processing is then manipulated by varying the nature of a horizontal string of letters that surrounds the target letter. Those letters are designated as "non-targets" (rather than distractors) because they are not response-eligible (e.g., they might be other letters than $\mathrm{X}$ or Z). A homogenous display of non-targets (e.g., "OOXOO") would be considered as representing a low-load condition, whereas a heterogeneous display (e.g., "WYXPK") would be considered a high-load condition. The canonical finding in this type of response competition paradigm (Lavie \& Tsal, 1994; Lavie \& Cox, 1997; Lavie, 1995, 2005), and replicated in attentional capture (Forster \& Lavie, 2008a,b) and in attentional blindness paradigms (Cartwright-Finch \& Lavie, 2007), is that the size of the distractor interference or congruency effect (the difference in response times for incongruent $>$ congruent distractors) is reduced, or even eliminated, under high as compared to low perceptual load conditions.

While perceptual load theory has the potential to reconcile mixed findings of early versus late selection effects in the literature, the model has been challenged. On the one hand, some researchers have argued that the theory is unsatisfactory because it offers no a priori, objective definition of what constitutes low or high perceptual load, and because it relies heavily on the vague notion of a limited resource (Benoni \& Tsal, 2013; Giesbrecht, Sy, Bundesen, \& Kyllingsbæk, 2014; Torralbo \& Beck, 2008). On the other hand, Benoni and Tsal (2010) have proposed a rival account for perceptual load effects on the basis of distractor dilution effects: here, it is argued that, for example, the heterogeneous letter strings in the high-load condition described above, rather than soaking up perceptual-attentional processing capacity in the course of target processing, are instead attentionally diluting the processing of the distractor stimulus, and are thus lessening its effectiveness in interfering with target processing. This distractor dilution interpretation is derived from the early visual interference account proposed by Brown, Roos-Gilbert, and Carr (1995) to explain the Stroop dilution phenomenon (Kahneman \& Chajczyk, 1983), whereby the additional presentation of a color-neutral word can reduce the interference from an incongruent color-word on color naming responses. Note that while both the load and dilution accounts refer to perceptual processing, in practice, many studies assessing these accounts have varied processing load in ways that go beyond purely manipulating perceptual difficulty, which is an issue we return to in the General discussion.

A typical dilution control experiment adopts the high-load condition of a perceptual load study (e.g., the letters $\mathrm{Z}$ or $\mathrm{X}$ surrounded by heterogeneous non-target consonants), but renders the target stimulus highly salient (e.g., color the target letter in bright red), such that the selection of the target is not perceptually difficult (low load) but the heterogeneous non-targets would still compete with the distractor. Importantly, it has been shown that under these dilution conditions, distractor interference is nevertheless highly reduced or eliminated (e.g., Tsal \& Benoni, 2010; Neokleous, Avraamides, Neocleous, \& Schizas, 2011; Wilson, Muroi, \& MacLeod, 2011). Thus, the same phenomenon-reduced distractor interference in the presence of heterogeneous compared to homogeneous non-targets - is explained by perceptual load theory as reflecting a lack of spare capacity for distractor processing due to high perceptual load in identifying the target, but by the dilution hypothesis as reflecting increased dilution of distractor processing by the non-targets. The overarching goal of the present study is to contrast the ability of these two accounts to explain some well-known empirical exceptions to the basic finding of reduced distractor interference under high perceptual load, as laid out below.

Lavie et al. (2003) measured interference from a horizontally flanking distractor face upon the classification of a famous name embedded among several letter strings above and below that name. Participants were required to categorize the famous name while ignoring the distractor face in the periphery, which could be congruent or incongruent with the target name. The level of perceptual load was manipulated by varying the target search set size (i.e., the number of non-target letter strings). Although mean response time increased significantly with search set size, this load manipulation did not affect interference effects from distractor faces (see also Jenkins et al., 2003; He and Chen, 2010), thus violating the standard load effect. However, these findings can be reconciled with the perceptual load model by adding an assumption that face processing relies on a specialized system whose capacity was unaffected by the level of load in a non-face search 
task (using written names as the search stimuli) (Lavie et al., 2003). However, Roberts and Besner (2005) also found that dilution effects occurred when non-targets were from the same "domain" (words or non-words) as distractors, but not when they were from different domains (e.g., Arabic digits), suggesting that the similarity in the level to which non-target and distractor stimuli are processed (e.g., perceptual vs. lexical vs. conceptual levels) might be an important determinant in how much they interfere with each other. We will here refer to this idea as the "processing overlap"-dependent interference or dilution. Accordingly, the interference effect observed at high load in Lavie et al. (2003) and similar studies could stem from a lack of dilution of face distractor processing by the real words or real names, consistent with the dilution account. However, due to a lack of dilution control conditions and/or a systematic manipulation of non-target-distractor processing overlap in previous studies, it is presently not clear whether the failure of high load to reduce interference from famous face distractors is due to specific resource consumption (in line with load theory) or dilution effects (in line with the dilution model), or a mixture of the two.

To adjudicate between these hypotheses, we conducted four experiments across which we modified the name classification task of Lavie et al. (2003) by introducing a dilution condition and manipulating the processing overlap between non-target and distractor stimuli. Specifically, as in Lavie et al. (2003), on each trial of the current experiments, one of six names of famous people (three statesmen and three pop stars) was presented (here, in Chinese characters) in a central column of the display, along with a varying number of non-targets. In addition, a distractor famous face stimulus was presented in the periphery, which could be congruent or incongruent with the target name. Subjects were required to decide whether the name belonged to a statesman or to a pop star. Thus, in order to complete the task, the target names had to be processed to a semantic or conceptual level (deciding whose name is shown). Moreover, the fact that distractor faces cause interference in this task (e.g., Lavie et al., 2003) indicates that those faces also elicit conceptual processing, presumably leading to the retrieval of the name and occupation of the famous person who's face is shown. In the low-load condition, the target name appeared by itself in one of several possible central column positions (i.e., varying along the vertical axis). In the high-load condition, the target was embedded among six non-targets items populating the vertical column (i.e., being presented above and/or below the target).

Crucially, we added the following two manipulations to this design: First, in an additional dilution condition, the central column display contained as many non-targets as in the high-load condition, but now the target was rendered highly distinguishable from these non-targets by being colored bright red, thus creating a situation of low perceptual demands on target processing but high potential for non-targets to dilute distractor processing (cf. Benoni \& Tsal, 2010; Tsal \& Benoni, 2010). This allowed us to assess the possible role of dilution in bringing about the effects observed in Lavie et al. (2003).

Secondly, the current study also systematically manipulated the processing level of the non-target stimuli, while keeping target and distractor stimuli constant, thus varying the degree in the processing overlap between non-targets and distractors/targets. For this manipulation, we capitalized on the fact that we employed Chinese characters as non-target stimuli, which allowed us to create (a) pseudo-characters that can be processed perceptually but are non-lexical and have no meaning; (b) random strings of actual Chinese characters that each allow for lexical processing but do not form a meaningful name, thus preventing conceptual-level processing; and (c) actual Chinese characters arranged in the proper order to form real names, allowing for conceptual processing (the non-target names contain common family names and first names in Chinese, which are also real words with semantic meanings). Thus, given that the targets and distractors are known to be processed up to the conceptual level (i.e., the persons' names and associated meaning), this manipulation gradually increased the degree of processing overlap between the nontargets and the distractors/targets, from the perceptual level to the lexical level to the conceptual level. By probing how the effects of this manipulation affected distractor interference effects in the low-load, high load and dilution conditions, respectively, we could determine whether the perceptual load theory or dilution accounts provide a more complete account of the determinants of distractor processing.

\section{Experiment 1}

In Experiment 1, we used a search plus classification task that was almost an exact replication of Lavie et al.'s (2003) study, except that the materials were modified to suit a Chinese sample, by replacing famous Western names and faces with famous Chinese ones, and English letter strings with Chinese pseudo-characters. Given this modified stimulus material and possible cultural differences, Experiment 1 had the sole goal of replicating the result of Lavie et al.'s (2003) in a Chinese sample, thus setting the stage for the planned modifications of the design in Experiments 2-4.

\section{Methods}

Sample size justification To achieve high power for detecting the impact of load/dilution manipulations on RT congruency effects, we used GPower (Faul et al., 2007) to estimate the effect size of the load by congruency interaction effect from a comparable previous paper (Tsal \& Benoni, 2010). Note that we could not employ Lavie et al. (2003) for this purpose, as the authors did not report any response time (RT) variance measures. The 
effect size of the interaction effect between congruency and load in the study of Tsal and Benoni (2010) was 0.42. With a desired power of 0.8 for detecting an effect at an alpha level of 0.05 , we would therefore need a minimum sample size of 15 . To ascertain high power, we set a target sample size of $\mathrm{N}=25$ for all experiments. Due to variability in participant scheduling reliability, we ended up with $\mathrm{N}=27$ in Experiment 1, $\mathrm{N}=23$ in Experiment 2, and $\mathrm{N}=25$ in Experiments 3 and 4.

Subjects Twenty-seven undergraduates from Southwest University (11 males; mean age $=22$ years, range $=19-26$ years) completed the experiment. They reported normal color vision and normal or corrected-to-normal acuity. This experiment, and the following three experiments, were all approved by the University Human Ethics Committee.

Stimuli and procedure The experiment was programmed and run using E-Prime. All stimuli were presented on a 15-in. computer screen at a viewing distance of $\sim 57 \mathrm{~cm}$. Each trial started with a 500-ms fixation point, followed by a 300- to 400-ms blank screen, after which the name-plus-face display was terminated by key press or after 2,000 ms. For all conditions, the name was equally likely to appear in any of the six vertical positions in the center of the screen, and the face was equally likely to appear to the left or right side of the central display (see Fig. 1). The face and name on a given trial were selected from a set of six politicians and six pop stars. The names were typed with Song Ti font size 12. Each face was placed in an imaginary rectangle that subtended $4.1^{\circ}$ by $3.3^{\circ}$

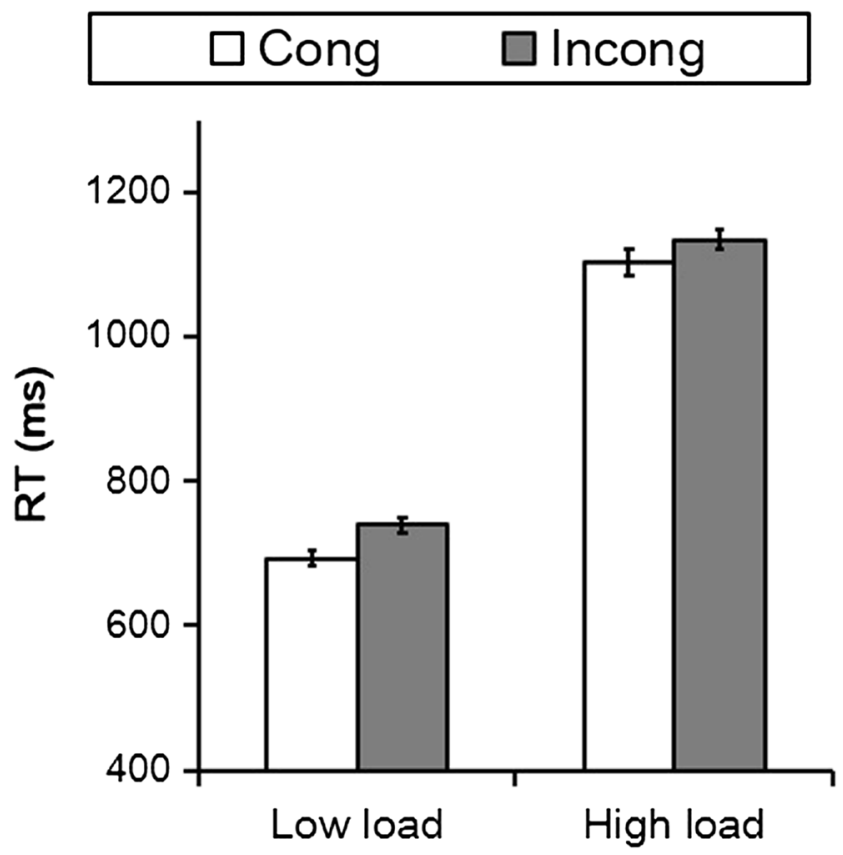

Fig. 1 Experiment 1 results. Mean response times (RTs) $( \pm$ SEM) for congruent (Cong) and incongruent (Incong) displays under low and high perceptual load conditions and was positioned with its center $5^{\circ}$ from fixation on the horizontal meridian. Subjects were required to search for a name among Chinese pseudo-characters in the center of a display and to indicate whether it was a politician's or a pop star's name by pressing the " 0 " or the " 2 " key on the numerical pad of the computer, respectively, while ignoring an irrelevant distractor face in the periphery. The mapping from name to key was counter-balanced across the participants. We manipulated the distractor's congruency with the target response. The distractor could be either the face of the person named (congruent condition) or the face of a person from the opposite category (incongruent condition). In the low-load condition, the target was presented alone, appearing randomly and equally likely in any one of the six horizontal positions. In the highload condition, the target was also presented randomly with equal probability in one of the six positions, but here five Chinese pseudo-characters appeared at the other five positions. Each subject ran through a practice block of 48 trials, followed by three experimental blocks of 64 trials each. Within each block, all conditions were randomly intermixed.

\section{Results and discussion}

The accuracy data of all four experiments are collectively presented in Table 1. However, because the mean accuracy is larger than $95 \%$ (near ceiling; see Table 1), we did not analyze the accuracy data and focused exclusively on response time analyses, as is typical in load experiments.

Incorrect responses and responses deviating by more than three $S D$ s from the mean were removed from the RT analyses. A 2 (distractor congruency: congruent vs. incongruent) $\times 2$ (load: low vs. high) within-subjects ANOVA performed on mean RT revealed that the two main effects were highly significant, due to slower RT for high compared to low-load conditions, $F(1,26)=1636.29, p<.001, \eta^{2}=.98$, and for incongruent compared to congruent distractors, $F(1,26)=$ $23.50, p<.001, \eta^{2}=.48$. There was no significant interaction of load and congruency, $F(1,26)=1.18, p>.28, \eta^{2}=.04$, thus indicating the lack of a classic load effect on distractor processing. Figure 1 presents the mean RTs as a function of the experimental variables.

The present data reflect a successful replication of Lavie et al. (2003)'s study with Chinese stimulus materials and a Chinese student sample. As in the original study, perceptual load had no effect on the extent to which distractor faces were processed: Distractor faces interfered with target performance at both low perceptual load and high perceptual load. It should be noted that, to make our results directly comparable with Lavie et al.'s (2003) study, we employed the identical design approach, which makes our results study optimally comparable to the original study but also means that our design inherited a couple of potential weaknesses from that study. First, the present measure of the interference effect - comparing incongruent 
Table 1 Mean percentage of correct responses (SDs are presented in parentheses) for Congruent (C) and Incongruent (I) trials in each condition, for each experiment

\begin{tabular}{|c|c|c|c|c|c|c|}
\hline \multirow[b]{2}{*}{ Congruency } & \multicolumn{2}{|l|}{ Low load } & \multicolumn{2}{|l|}{ High load } & \multicolumn{2}{|l|}{ Dilution } \\
\hline & $\mathrm{C}$ & I & $\mathrm{C}$ & I & $\mathrm{C}$ & I \\
\hline Experiment 1 & $0.97(0.03)$ & $0.97(0.04)$ & $0.92(0.06)$ & $0.93(0.04)$ & & \\
\hline Experiment 2 & $0.98(0.03)$ & $0.96(0.03)$ & $0.97(0.02)$ & $0.96(0.03)$ & $0.97(0.03)$ & $0.98(0.03)$ \\
\hline Experiment 3 & $0.97(0.04)$ & $0.94(0.05)$ & $0.93(0.05)$ & $0.94(0.05)$ & $0.97(0.03)$ & $0.96(0.04)$ \\
\hline Experiment 4 & $0.98(0.03)$ & $0.96(0.04)$ & $0.94(0.04)$ & $0.92(0.06)$ & $0.97(0.03)$ & $0.97(0.03)$ \\
\hline
\end{tabular}

RTs with congruent RTs - is arguably suboptimal because congruent trial RTs can be influenced both by attentional and nonattentional mechanisms (like redundancy gain). Second, the distractor face was identical to the target name in congruent trials whereas the distractor face was one of three faces from the opposite category (pop stars or politicians) in incongruent trials, which represents an asymmetry between compatible and incompatible trials with respect to identity versus categorical relatedness between the target and distractor. However, these suboptimal features in our design are equal across the main condition comparisons of interest and are present in all of our experiments and can therefore not account for differential results between experiments, and they replicate the approach of many prior studies in this field.

\section{Experiment 2}

Following the replication of Lavie et al.'s design and results in Experiment 1, we pursued two new aims in Experiment 2. First, we sought to rule out a possible alternative explanation for the presence of distractor interference in the high-load condition. Namely, a number of previous studies have indicated that while distractor interference under high load is observed when low and high-load trials are intermixed within a block, the standard load effects of reduced distractor interference can nevertheless emerge when low- and high-load conditions are presented in separate blocks (Benoni, Zivony, \& Tsal, 2013; Biggs \& Gibson, 2010; Cave \& Chen, 2016; Murray \& Jones, 2002; Theeuwes et al., 2004). Thus, considering the trial-mixing design in Lavie et al. (2003) and in the current Experiment 1, it was important to examine whether the interference from famous face distractors independent of perceptual load would also be observed when perceptual load conditions were blocked. Second, we added the dilution condition (coloring the target name in red) in order to probe whether that condition would mimic the results of the highload condition in the absence of an explanation based on high perceptual load (cf. Tsal \& Benoni, 2010). Specifically, we could examine whether, under blocked conditions, the interference effect would be modulated by the non-target dilution.
Notably, in addition to the overall 2 (congruency: congruent vs. incongruent) $\times 3$ (load: low vs. high vs. dilution) ANOVA, three follow-up $2 \times 2$ ANOVAs were also conducted, where the first variable was congruency and the second variable was load condition. For the congruency variable, there were always two levels: congruent versus. incongruent. However, the levels of load variable varied from low versus high to low versus dilution to high versus dilution, by which the influences of perceptual load and dilution on the interference effect could be examined in detail. The comparison between low-load and high-load conditions' effect on distractor interference assesses the traditional perceptual load effect, but is notably confounded with respect to dilution, as the low-load condition has both a low perceptual load and no dilution, whereas the high-load condition has both high perceptual target load and is high in dilution. Therefore, the results of that test have to be interpreted in the light of the other two followup ANOVAs. Specifically, the comparison between the low load and dilution conditions' effect on distractor interference isolates the effect of distractor dilution, as both conditions have low target processing load but vary with respect to dilution (no dilution for the low-load condition, high dilution for the dilution condition). Finally, the comparison between the high load and dilution conditions' effect on distractor interference isolates the effect of difficulty (i.e., load) in perceptual target processing, as both conditions are high in dilution but differ in target load (low in the dilution condition but high in the high-load condition. The three follow-up $2 \times 2$ ANOVAs were included in the current and following two experiments.

\section{Methods}

Subjects Twenty-three undergraduates from Southwest University (five males; mean age $=21$ years, range $=19-24$ years) completed the experiment. They reported normal color vision and normal or corrected-to-normal acuity. For sample size justification, see Experiment 1.

Stimuli and procedure The apparatus and procedure were identical to those in Experiment 1, except that three experimental conditions (low-load, high-load and dilution) were 
included and presented in separate blocks rather than being mixed together within blocks. An equal number of trials in each block type included congruent versus incongruent distractor stimuli. In the dilution condition, the displays were identical to those of the high-load condition, except that the target name was presented in red (see Fig. 2). The subjects were presented with the different conditions in different blocks of trials. Three blocks of 96 trials each were presented in a random order and counter-balanced over subjects. Before the formal experiment, each subject ran through a practice block of 48 trials.

\section{Results and discussion}

The accuracy data are presented in Table 1. Incorrect responses and responses deviating by more than $3 S D$ s from the mean were removed from the RT analyses. A 2 (distractor congruency: congruent vs. incongruent) $\times 3$ (load: low vs. high vs. dilution) within-subjects ANOVA on RT revealed that the two main effects were highly significant, $F(2,44)=$ 907.72, $p<.001, \eta^{2}=.98$ for load condition; $F(1,22)=$ $76.60, p<.001, \eta^{2}=.78$ for congruency. As in Experiment 1 , there was no significant interaction observed between load condition and congruency, $F(2,44)=.49, p>.61, \eta^{2}=.02$. Figure 3 presents mean RTs for all conditions.

In sum, even in a design that blocked low- and high-load (and dilution) conditions, perceptual load had no effect on the extent to which distractor faces were processed; as in previous studies (He \& Chen, 2009; Lavie et al., 2003), distractor faces interfered with target performance, even under high load. Moreover, the same was true for the dilution condition. These results indicate that neither making the target harder to distinguish (high-load condition) nor diluting the distractor with heterogeneous non-targets (dilution condition) reduces distractor interference, suggesting that the interference from famous face distractors is rather robust. Notably, however, the non-targets were Chinese pseudo-characters, which can be assumed to have little processing overlap with the distractor and target stimuli.

\section{Experiment 3}

The results of Experiment 2 do not allow us to differentiate between the "spare resource hypothesis" (load theory) and the distractor dilution hypothesis (dilution theory). To adjudicate between the two, in Experiments 3 and 4, we increased the degree of processing overlap between non-targets and targets/ distractors, by gradually making them more similar. In Experiments 1 and 2, the non-target stimuli were Chinese pseudo-characters, which are categorically distinct from proper names and faces, such that the shared-resource consumption or distractor dilution would be expected to be low (e.g., Benoni \& Tsal, 2010; Kahneman \& Chajczyk, 1983; Wilson et al., 2011). To increase similarity, in Experiment 3 the Chinese pseudo-characters were replaced by random strings of real Chinese characters. Importantly, these can be expected to evoke lexical processing, but do not represent a meaningful name with semantic or conceptual connotations. Under the

\section{Low load}
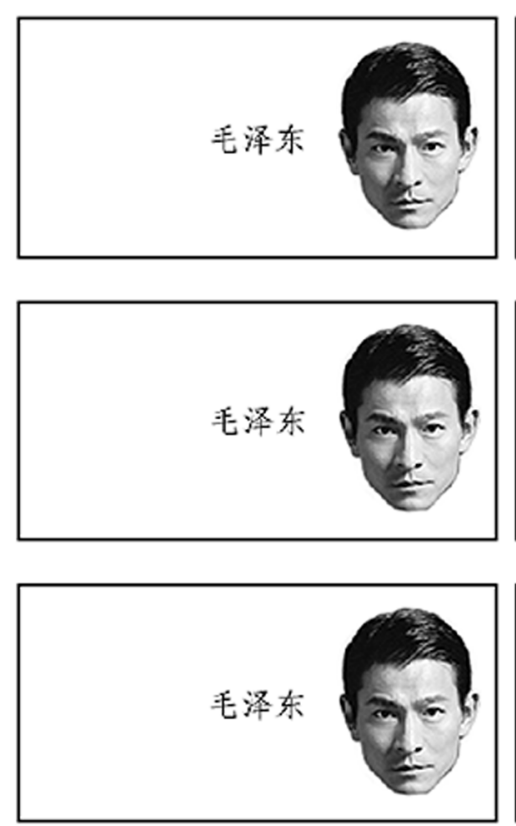

Fig. 2 Example stimulus displays. The first, second, and third rows show example stimuli used in Experiments 2, 3, and 4, respectively. For all the conditions, the target name was equally likely to appear in any of the six
High load
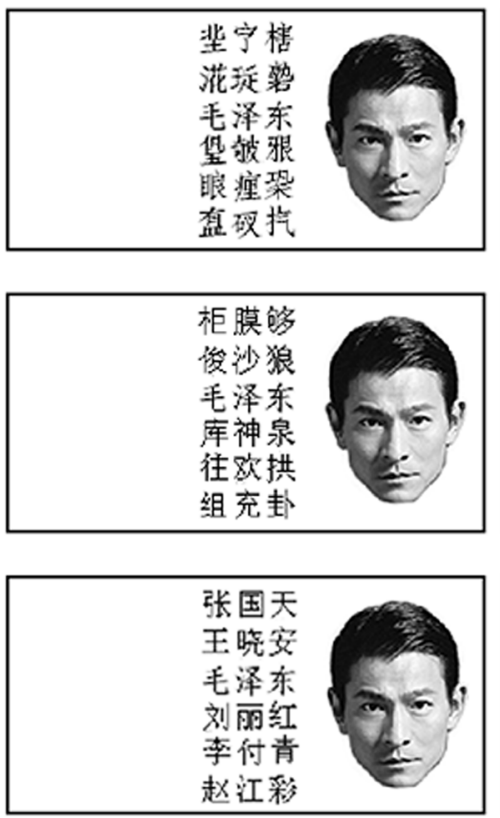

Dilution
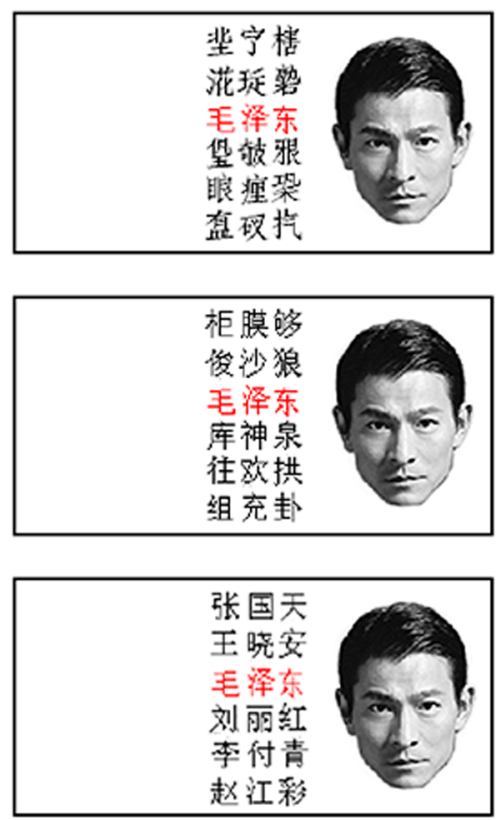

vertical positions, and the distractor face was equally likely to appear on the left or right 


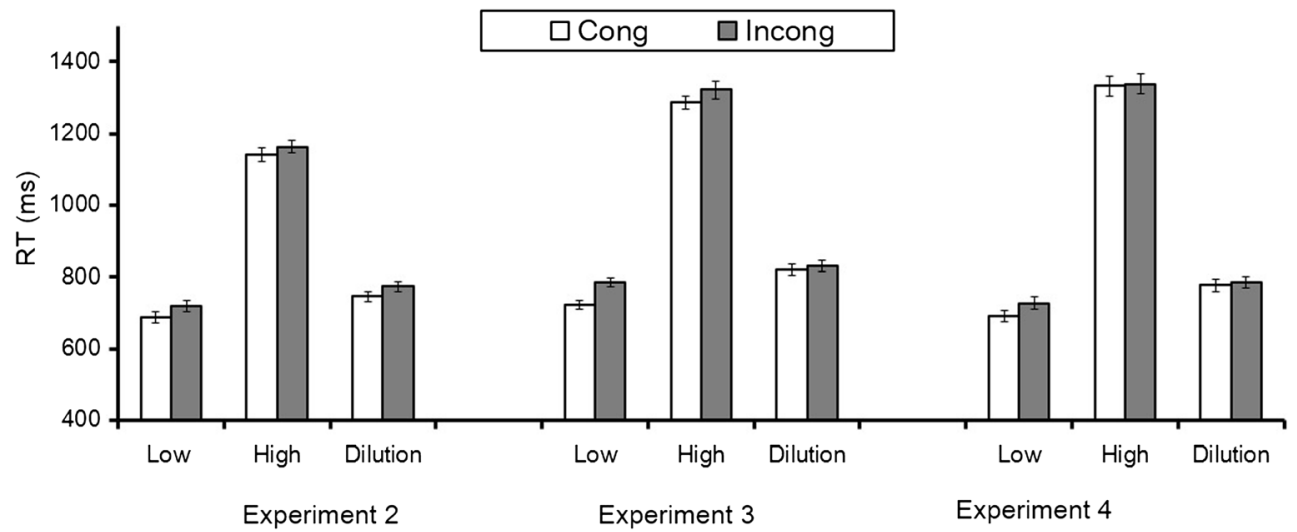

Fig. 3 Mean response times (RTs) ( \pm SEM) for congruent (Cong) and incongruent (Incong) distractor conditions under low-load, high-load, and dilution conditions for Experiments 2, 3, and 4

assumption that in conditions involving non-target stimuli, greater overlap in processing level would be associated with a greater dilution effect, we could then test whether that effect was present in the high perceptual load condition exclusively, or would also be observed in the dilution condition.

\section{Methods}

Subjects Twenty-five undergraduates from Southwest University (eight males; mean age $=22$ years, range $=19$ 25 years) completed the experiment. They reported normal color vision and normal or corrected-to-normal acuity. For sample size justification, see Experiment 1.

Stimuli and procedure All stimuli, apparatus, and procedures were identical to those in Experiment 2 with the exception that, in the high-load and dilution conditions, the target name was accompanied by non-targets comprised of real Chinese words (see Fig. 2).

\section{Results and discussion}

The accuracy data are presented in Table 1. Responses deviating by more than $3 S D$ s from the mean and incorrect response trials were removed from the RT analyses. A 2 (distractor congruency: congruent vs. incongruent) $\times 3$ (load: low vs. high vs. dilution) within-subjects ANOVA performed on RTs revealed that the two main effects and their interaction were all significant, $F(2,48)=1191.21, p<.001, \eta^{2}=.98$ for load condition; $F(1,24)=19.78, p<.001, \eta^{2}=.45$ for congruency; and $F(2,48)=4.30, p<.05, \eta^{2}=.15$ for the interaction. Figure 3 shows mean RTs for congruent and incongruent distractors under the three load conditions.

Three follow-up $2 \times 2$ ANOVAs were carried out to reveal the separate effects of load and dilution. First, a 2 (congruency: congruent vs. incongruent) $\times 2$ (load: low vs. high) ANOVA revealed that RTs were faster in the low-load condition than in the high-load condition, $F(1,24)=1431.30, p<$
$.001, \eta^{2}=.98$, and congruent displays were responded to faster than incongruent ones, $F(1,24)=24.08, p<.001, \eta^{2}$ $=.50$. As in Experiment 1, the interaction between these two factors was not significant, $F(1,24)=1.75, p>.19, \eta^{2}=.07$. Effects of congruency were significant at low load $(p<.001)$ and approached statistical significance at high load $(p=.07)$. Thus, the traditional perceptual load effect on distractor interference, which confounds load and dilution, was not observed.

The second $2 \times 2$ ANOVA crossed the congruency factor with a load factor involving the low-load and dilution conditions to selectively assess the effect of dilution on distractor interference. The analysis showed that congruent displays were responded to faster than incongruent ones, $F(1,24)=$ $30.17, p<.001, \eta^{2}=.56$, and that the target was identified faster in the low load than dilution condition, $F(1,24)=$ $58.75, p<.001, \eta^{2}=.71$. Most importantly, the interaction between load condition and congruency was also significant, $F(1,24)=14.74, p<.01, \eta^{2}=.38$, due to greater interference under the low-load condition than under the dilution condition. Moreover, in the dilution condition, the interference was completely eliminated, as congruent and incongruent displays were responded to equally fast, $F(1,24)=.88, p>.35, \eta^{2}=$ .03 . This finding, paired with a lack of a similar effect in the high-load condition, strongly suggests that distractor interference is determined by dilution effects of non-targets on distractors rather than by perceptual target load.

Third, a $2 \times 2$ ANOVA crossing the congruency factor with a load factor involving the high-load and dilution conditions assessed any effects that high perceptual load may have above and beyond the dilution effect. This analysis showed that the target was identified faster in the dilution condition than in the high-load condition, $F(1,24)=$ $1514.68, p<.001, \eta^{2}=.98$, but neither the main effect of congruency, $F(1,24)=3.80, p=.06, \eta^{2}=.14$, nor the interaction were significant, $F(1,24)=1.82, p>.19, \eta^{2}=$ .07 . Effects of congruency approached statistical significance in the high-load condition $(p=.07)$ but were completely eliminated in the dilution condition $(p>.36)$. 
Taken together, the results of Experiment 3 indicate that when non-targets have some processing overlap with targets/ distractors, interference from incongruent distractors can be eliminated through non-targets diluting the distractor's influence, but not through high perceptual target load. The mean RT in the high-load condition in Experiment 3 was significantly higher than that in Experiment $2(F(1,22)=25.77, p<$ $\left..001, \eta^{2}=.54\right)$, suggesting that the processing overlap between non-targets and targets/distractors was indeed enhanced. The enhanced overlap should enhance the effect of perceptual load according to the "spare resources hypothesis" (load theory) and the effect of distractor dilution according to the distractor dilution hypothesis. The absence of interference in the dilution condition clearly supports the dilution hypothesis. By contrast, the presence of an interference effect in the high-load condition when the effect of perceptual load is enhanced speaks against the "spare resources hypothesis." It is somewhat surprising that even though dilution eliminated the interference effect when target-processing load was low (dilution condition), high load appeared to counteract this effect, with a marginal interference effect observed in the high-load (also high-dilution) condition. We are not sure as to the source of this data pattern, but Experiment 4, in addition to probing the effect of further increasing the processing overlap between non-targets and distractors/targets, also allowed us to probe whether this surprising aspect of the results of Experiment 3 would prove replicable. In Experiment 4, we further enhanced the processing overlap between non-targets and targets/ distractors by utilizing real Chinese names (which are also real Chinese words) as non-targets.

\section{Experiment 4}

Experiments 2 and 3 demonstrated that face-name interference could be diluted when the non-target stimuli were real Chinese characters, but not when the non-target stimuli were Chinese pseudo-characters. In Experiment 4, we sought to replicate this finding while testing whether an even greater processing overlap between non-targets and distractors/targets (here, at the conceptual level) would further enhance these effects. In Experiment 4, therefore, distractor congruency and load were manipulated as before, and all other aspects of procedure were the same as the procedures in Experiments 2 and 3, except that in order to increase overlap processing level from the lexical to the conceptual domain, here the random strings of Chinese characters were replaced by common family names and first names in Chinese, which also have semantic meaning.

\section{Methods}

Subjects Twenty-five undergraduates from Southwest University ( six males; mean age $=22$ years, range $=18-24$ years) completed the experiment. They reported normal color vision and normal or corrected-to-normal acuity. For sample size justification, see Experiment 1.

Stimuli and procedure All stimuli, apparatus, and procedures were identical to those in above two experiments with the exception that subjects were required to search for one of six famous names among other proper Chinese names in the center of a display in the high load and dilution conditions (see Fig. 2).

\section{Results and discussion}

The accuracy data are presented in Table 1. Responses deviating by more than $3 \mathrm{SDs}$ from the mean and incorrect responses were removed from the RT analyses. A 2 (distractor congruency: congruent vs. incongruent) $\times 3$ (load: low vs. high vs. dilution) within-subjects ANOVA carried out on mean RTs showed that both main effects and their interaction were significant: $F(2,48)=995.96, p<.001, \eta^{2}=.98$ for load condition; $F(1,24)=7.95, p<.01, \eta^{2}=.25$ for congruency; and $F(2,48)=3.69, p<.05, \eta^{2}=.13$ for their interaction. Figure 3 shows mean RTs for congruent and incongruent distractors under the three load conditions.

Three follow-up $2 \times 2$ ANOVAs were carried out to reveal the separate effect of load and dilution. First, a 2 (congruency: congruent vs. incongruent) $\times 2$ (load: low vs. high) ANOVA assessed the traditional perceptual load effect. All effects were significant; for load condition, $F(1,24)=1278.15, p<.001$, $\eta^{2}=.98$; for congruency, $F(1,24)=6.63, p<.05, \eta^{2}=.22$; and for their interaction, $F(1,24)=4.30, p<.05, \eta^{2}=.15$. Analyses of simple effects showed that the congruency effect was significant under the low-load condition, $F(1,24)=$ 22.33, $p<.001, \eta^{2}=.48$, and completely eliminated at the high-load condition, $F(1,24)=.15, p>.70, \eta^{2}=.01$. Thus, in the presence of high overlap in processing level between nontargets and distractor and target stimuli, the traditional (albeit confounded) perceptual load effect, mixing is observed.

The second $2 \times 2$ ANOVA crossed the congruency factor with a load factor involving the low load and dilution conditions in order to isolate the effect of dilution on distractor interference. Again, all effects were significant, $F(1,24)=$ $112.43, p<.001, \eta^{2}=.82$ for load condition; $F(1,24)=20.93$, $p<.001, \eta^{2}=.47$ for congruency; and $F(1,24)=11.99, p<$ $.01, \eta^{2}=.33$ for their interaction. Analyses of simple effects indicated that while the congruency effect was highly significant for the low-load condition, $F(1,24)=22.33, p<.001, \eta^{2}$ $=.48$, it was completely eliminated in the dilution condition, $F$ $(1,24)=2.39, p>.13, \eta^{2}=.09$. Thus, as in Experiment 3, we observed a distractor dilution effect.

Third, a $2 \times 2$ ANOVA crossed the congruency factor with a load factor involving the high-load and dilution conditions in order to isolate the pure effect of perceptual load. Here, only 
the main effect of load was highly significant, $F(1,24)=$ $867.70, p<.001, \eta^{2}=.97$. The main effect of congruency was not significant, $F(1,24)=.72, p>.40, \eta^{2}=.03$, and it also did not interact with load condition, $F(1,24)=.02, p>$ $.88, \eta^{2}=.001$. Thus, perceptual load per se was not capable of reducing distractor interference in Experiment 4.

With greater processing overlap between non-targets and targets/distractors, the distractor interference effect was eliminated in both the high-load and dilution conditions in Experiment 4, unlike in Experiment 3, where this was only the case in the dilution condition. Moreover, the mean RT in the high-load condition in Experiments 3 was not significantly longer than that in Experiment $4\left(F(1,24)=.61, p>.44, \eta^{2}=\right.$ $.03)$, suggesting that the perceptual load at high load was not enhanced in Experiment 4, relative to that in Experiment 3. Thus, the source of the interference elimination in Experiment 4 was distractor dilution rather than perceptual target load, as shown by the absence of interference at high load, as well as the significant dilution effect when comparing the dilution condition against the low-load condition. These findings provide further support for the processing overlap dependent dilution account and speak against the perceptual load account. In sum, as the processing overlap between non-targets and distractors/targets was further enhanced through using real names as non-targets, the results showed that distractor dilution could eliminate the interference from famous face distractors, whereas perceptual load could not.

\section{General discussion}

In the present study, we sought to evaluate the ability of perceptual load and dilution accounts to explain distractor interference effects in the context of a well-known exception to typical perceptual load effects, namely the failure of reducing famous face distractor interference in high-load name target conditions (He and Chen, 2010; Lavie et al., 2003). This finding has previously been reconciled with load theory by arguing for (face-)specific perceptual attention resources. By adding a distractor dilution condition and manipulating the processing overlap between non-targets and distractor, we here evaluated whether the failure of high load to reduce interference from famous face distractors is in fact due to specific resource consumption (in line with load theory) or alternatively due to a dilution account, or a mixture of the two. Experiment 1 replicated the original effect of no interference modulation (Lavie et al., 2003), but using Chinese stimulus materials in a Chinese sample. Experiment 2 again replicated this effect, but with blocked load conditions (cf. Benoni, Zivony, \& Tsal, 2013; Biggs \& Gibson, 2010; Cave \& Chen, 2016; Murray \& Jones, 2002; Theeuwes et al., 2004), and showed that with little overlap in processing level between non-targets and distractors, no dilution effects are observed. Crucially, Experiments 3 and 4 gradually increased that overlap, moving from perceptual (Experiments 1 and 2) to lexical (Experiment 3) to conceptual (Experiment 4) non-target processing, and showed that this manipulation reintroduces modulation of interference effects but, crucially, that this modulation was attributable to distractor dilution rather than perceptual load effects. We discuss these findings in turn.

The first two experiments replicated previous findings of no-load effects on distractor interference when the processing overlap between non-targets and distractors was low in mixedload conditions, and extended previous work by showing that the same is also true for blocked-load conditions. Thus, the interference from famous faces when categorizing famous names is rather robust, generalizing across different cultures, stimulus materials, and blocking conditions. The latter finding is important as it ruled out an alternative account for the original effect, namely that load effects on interference are sometimes exclusively observed under blocked-load conditions (Benoni, Zivony, \& Tsal, 2013; Biggs \& Gibson, 2010; Cave \& Chen, 2016; Murray \& Jones, 2002; Theeuwes et al., 2004). Finally, Experiment 2 also confirmed that under low overlap in processing level between non-targets and distractors, neither perceptual load nor distractor dilution effects are observed. Experiments 1 and 2 thus provided the setting for directly comparing how perceptual load versus dilution conditions would impact on distractor interference once the processing overlap between non-targets and distractors (and targets) was increased.

We manipulated the processing overlap by changing nontarget stimuli from Chinese pseudo-characters (perceptual processing level, Experiments 1 and 2) to real Chinese characters arranged in random order (lexical processing level, Experiment 3) to real Chinese characters arranged in the proper order to form real Chinese names (conceptual processing level, Experiment 4). The elimination of the interference effect in the dilution conditions of Experiments 3 and 4 confirmed the dilution effect. Tsal and Benoni (2010), in proposing the dilution account argued that when non-targets are added in the high-load or dilution displays, their features compete with those of the incongruent distractor and degrade the quality of its visual representation, thus substantially reducing the amount of lexical analysis performed on the distractor. This, in turn, attenuates the activation of the (distractor-related) incongruent response category, thereby reducing or eliminating the congruency effect. Our findings show that the distractor interference was affected by the processing overlap between non-targets and distractor, that is, the interference effect in the dilution condition was eliminated when the depth of processing of non-targets and distractor became more similar (in the present study, from perceptual to lexical to conceptual processing).

Specifically, in the present study design, for interference effects to occur between the distractor face and the target name (as in, e.g., Experiment 1), the distractor face has to be 
processed to a conceptual level, thus producing a mismatch between the person denoted by the target name and the person shown as the distractor. Based on this, we reasoned that the more similar the processing level of the non-targets gets to the level of conceptual processing, the more the non-targets should be able to dilute distractor processing, and this motivated the designs of Experiments 3 and 4.

When non-targets are pseudo-Chinese characters (in Experiment 2), the processing of non-targets is restricted to the perceptual level, because the characters are meaningless. Thus, there is little overlap in the processing level between non-targets and the distractor, and there is no dilution effect. When moving from non-target pseudo-characters to random strings of real characters (in Experiment 3), the non-targets should engage lexical (but not conceptual) processing, because while each of the three Chinese characters has its own meaning, a random arrangement of three such characters is meaningless and does not result in a conventional name with conceptual implications. In Experiment 3, the eliminated interference effect in the dilution condition indicated that the real-word non-targets can dilute the distractor face to a certain extent (lexical level). However, the still significant interference effect in the high-load condition of Experiment 3 may result from the fact that the high perceptual load results in a substantial increase in processing time, which in turn, increases the probability of irrelevant intrusion (Tsal \& Benoni, 2010; Wilson et al., 2011). The results of Experiment 3 indicated that the dilution effect of non-targets can occur when non-target are processed up to the lexical level. In Experiment 4, the non-targets were real Chinese names, which can be expected to evoke conceptual-level processing, thus creating a condition of greatest overlap with the processing of the distractor. Accordingly, we observed a complete elimination of the interference effect in the dilution condition in Experiment 4.

The changes from pseudo-Chinese characters to randomly arranged real Chinese characters to real Chinese names reflect a systematic, gradual change in the level/depth of processing, from perceptual to lexical to conceptual. Our experimental manipulations can thus distinguish how changes in the processing overlap between non-targets and distractors drive dilution effects. Consequently, we put forward a processing overlap explanation that argues that the degree of dilution increases with the increasing processing overlap (or similarity) between nontargets and distractor, and reaches its maximum when the two processing levels match (Experiment 4: conceptual-level processing). An important remaining question in this regard is to what degree the present results are purely driven by variation in the processing overlap between non-targets and distractors versus the concomitant variation in overlap between the nontargets and the target stimulus. Creating a scenario where these two overlaps can be manipulated independently represents an intriguing challenge for future studies.

Results that are compatible with the present findings have also been reported in the context of Stroop (Roberts \& Besner,
2005) and Simon effect dilution studies (Miles, Yamaguchi, \& Proctor, 2009). For instance, Miles and colleagues (2009) in a word-based Simon task found that a word diluter reduced the compatibility effect produced by a location-word distractor but not that produced by a spatial symbol distractor, thus providing evidence that stimulus-response compatibility effects were susceptible to the presence of diluters that were categorically similar to the distractors. Both the current results and those previous studies clearly support the notion that the degree to which incidental stimuli (non-targets) share conceptual or perceptual features with target-incongruent distractors determines the amount of distractor dilution (cf., Tsal \& Benoni, 2010).

Our findings are also compatible with those of a recent study by Biggs and Gibson (2014), who reported that manipulating the salience of the distractor can modulate interference effects independent of dilution and load effects. Finally, Kyllingsbæk, Sy, and Giesbrecht (2011) applied a version of the theory of visual attention (TVA) to modeling load effects, whereby attention is allocated in a single step over all stimuli, in proportion to their respective "attentional weights" rather than in a two-step process advocated by perceptual load theory, where attention is initially allocated to relevant stimuli, and in case of remaining capacity spills over to irrelevant stimuli (see also Giesbrecht et al., 2014). The present results do not contradict this alternative explanation for load or dilution effects, though our experimental protocol is sufficiently distinct from Kyllingsbæk et al. (2011), who employed single letters rather than words and faces as stimuli, that a reinterpretation of the current data within this TVA account would require a lot of uncertain assumptions. However, an extension of a TVA perspective to load experiments employing word stimuli would represent an interesting target for future modeling work.

Finally, two important conceptual points merit discussion. First, as briefly noted in the introduction, both load and dilution theory have been originally formulated in terms of perceptual processing resources. However, the present experiments, as well as many others in this field (e.g., Lavie et al., 2003; Roberts \& Besner, 2005), have clearly expanded the conceptions of load and dilution effects to manipulating task factors that are not perceptual but conceptual in nature. Thus, the current results not only suggest that a dilution account serves as a better explanation for the drivers of attentional selectivity than the load account, but also that the dilution account needs to be formally expanded to incorporate nonperceptual processing domains.

Second, a key drawback of load theory and the associated experimental protocol (which we adopted here) is an inherent ambiguity as to how distracter interference under high-load (large target set size) conditions should be interpreted. One common assumption in load theory is that if distracter interference occurs in the presence of high perceptual load, this would represent evidence for distracter processing being 
automatic (i.e., not requiring attention). An alternative interpretation, as pursued by Lavie et al. (2003), is to interpret such effects as being related to distinct pools of attentional resources for different types of stimulus materials, rather than distracter processing requiring no attention at all. Accordingly, Benoni (2018) has recently put forward a number of cogent arguments against interpreting results of the standard load theory task design, where target set size is the primary manipulated factor, in terms of the relative automaticity of distracter processing. Moreover, it has been noted that many studies that have produced supposedly supportive evidence for automatic distracter processing within the load theory framework can be criticized for succumbing to a circular logic (Lamy, Leber, \& Egeth, 2013): they interpret results from load manipulations under the assumption that load theory is correct in the first place. These inherent weaknesses in the most prominent experimental approach to studying the determinants of attentional selection should motivate researchers to pursue new, less ambiguous means for assessing the effects of processing load and dilution in future studies.

In conclusion, we demonstrated that interference effects from distractors decrease with an increase in overlap in the processing level between additional non-targets and the distractor. Importantly, however, that interference is reduced (and at times eliminated) by distractor dilution rather than by perceptual target load. Together, these data are incompatible with a perceptual load account of attentional selection but they support a processing overlap dependent distractor dilution account.

Acknowledgements This work was supported by the National Natural Science Foundation of China $(61431013,31771254)$, and the Fundamental Research Funds for the Central Universities (SWU1609318, SWU1609106, SWU1709107).

\section{References}

Benoni, H. (2018). Can automaticity be verified utilizing a perceptual load manipulation? Psychonomic Bulletin \& Review, doi:https:// doi.org/10.3758/s13423-018-1444-7

Benoni, H., \& Tsal, Y. (2010). Where have we gone wrong? Perceptual load does not affect selective attention. Vision Research, 50, 12921298. doi:https://doi.org/10.1016/j.visres.2010.04.018

Benoni, H., \& Tsal, Y. (2013). Conceptual and methodological concerns in the theory of perceptual load. Frontiers in Psychology, 4, 522. doi: https://doi.org/10.3389/fpsyg.2013.00522

Biggs, A. T, \& Gibson, B. S. (2010). Competition between color salience and perceptual load during visual selection can be biased by topdown set. Attention, Perception, \& Psychophysics, 72, 53-64. doi: https://doi.org/10.3758/APP.72.1.53

Biggs, A. T., \& Gibson, B. S. (2014). Visual salience can co-exist with dilution during visual selection. Journal of Experimental Psychology: Human Perception and Performance, 40, 7-14. doi: https://doi.org/10.1037/a0033922

Broadbent, D. (1958). Perception and communication. London: Pergamon Press.
Brown, T. L., Roos-Gilbert, L., \& Carr, T. H. (1995). Automaticity and word perception: Evidence from Stroop and Stroop dilution effects. Journal of Experimental Psychology: Learning, Memory, and Cognition, 21, 1395-1411. doi:https://doi.org/10.1037/0278-7393. 21.6.1395

Cartwright-Finch, U., \& Lavie, N. (2007). The role of perceptual load in inattentional blindness. Cognition, 102, 321-340. doi:https://doi. org/10.1016/j.cognition.2006.01.002

Cave, K. R., \& Chen, Z. (2016). Identifying visual targets amongst interfering distractors: Sorting out the roles of perceptual load, dilution, and attentional zoom. Attention, Perception, \& Psychophysics, 78, 1822-1838. doi:https://doi.org/10.3758/s13414-016-1149-9

Deutsch, J. A., \& Deutsch, D. (1963). Attention: Some theoretical considerations. Psychological Review, 70, 80-90. doi:https://doi.org/10. 1037/h0039515

Faul, F., Erdfelder, E., Lang, A.-G., \& Buchner, A. (2007). G*Power 3: A flexible statistical power analysis program for the social, behavioral, and biomedical sciences. Behavior Research Methods, 39, 175-191

Forster, S., \& Lavie, N. (2008a). Attentional capture by entirely irrelevant distractors. Visual Cognition, 16, 200-214. doi:https://doi.org/10. 1080/13506280701465049

Forster, S., \& Lavie, N. (2008b). Failures to ignore entirely irrelevant distractors: The role of load. Journal of Experimental Psychology: Applied, 14, 73-83. doi:https://doi.org/10.1037/1076-898X.14.1.73

Giesbrecht, B., Sy, J., Bundesen, C., \& Kyllingsbæk, S. (2014). A new perspective on the perceptual selectivity of attention under load. Annals of the New York Academy of Sciences, 1316, 71-86. doi: https://doi.org/10.1111/nyas.12404

He, C., \& Chen, A. (2010). Interference from familiar natural distractors is not eliminated by high perceptual load. Psychological Research, 74, 268-276. doi:https://doi.org/10.1007/s00426-009-0252-0

Jenkins, R., Lavie, N., \& Driver, J. (2003). Ignoring famous faces: category-specific dilution of distractor interference. Perception \& Psychophysics, 65, 298-309. doi:https://doi.org/10.3758/ BF03194801

Kahneman, D., \& Chajczyk, D. (1983). Tests of the automaticity of reading: dilution of Stroop effects by color-irrelevant stimuli. Journal of experimental psychology: Human perception and performance, 9, 497-509. doi:https://doi.org/10.1037/0096-1523.9.4.497

Kyllingsbæk, S., Sy, J. L., \& Giesbrecht, B. (2011). Understanding the allocation of attention when faced with varying perceptual load in partial report: A computational approach. Neuropsychologia, 49, 1487-1497. doi:https://doi.org/10.1016/j.neuropsychologia.2010. 11.039

Lamy, D., Leber, A. B., \& Egeth, H. E. (2013). "Selective attention", in Handbook of Psychology, Experimental Psychology, 2nd Edn, Vol. 4 eds R.W. Proctor and I. B.Weiner (Hoboken, NJ: JohnWiley and Sons Inc), 267-294.

Lavie, N. (1995). Perceptual load as a necessary condition for selective attention. Journal of Experimental Psychology: Human Perception and Performance, 21, 451-468. doi:https://doi.org/10.1037/00961523.21.3.451

Lavie, N. (2005) Distracted and confused?: Selective attention under load. Trends in Cognitive Sciences, 9, 75-82. doi:https://doi.org/ 10.1016/j.tics.2004.12.004

Lavie, N. (2010). Attention, distraction, and cognitive control under load. Current Directions in Psychological Science, 19, 143-148. doi: https://doi.org/10.1177/0963721410370295

Lavie, N., \& Cox, S. (1997). On the efficiency of attentional selection: Efficient visual search results in inefficient rejection of distraction. Psychological Science, 8, 395-398.

Lavie, N., Ro, T., \& Russell, C. (2003). The role of perceptual load in processing distractor faces. Psychological Science, 14, 510-515. doi:https://doi.org/10.1111/1467-9280.03453 
Lavie, N., \& Tsal, Y. (1994). Perceptual load as a major determinant of the locus of selection in visual attention. Perception \& Psychophysics, 56, 183-197. doi:https://doi.org/10.3758/BF03213897

Miles, J. D., Yamaguchi, M., \& Proctor, B. W. (2009).Dilution of compatibility effects in Simon-type tasks depends on categorical similarity between distractors and diluters. Attention, Perception, \& Psychophysics, 71, 1598-1606. doi: https://doi.org/10.3758/APP. 71.7.1598.

Murray, J. E., \& Jones, C. (2002).Attention to local form information can prevent access to semantic information. The Quarterly Journal of Experimental Psychology: Section A, 55, 609-625. doi:https://doi. org/10.1080/02724980143000370

Neokleous, K., Avraamides, M. N., Neocleous, C. K., \& Schizas, C. N. (2011). Selective attention and consciousness: investigating their relation through computational modeling. Cognitive Computation, 3, 321-331. doi:https://doi.org/10.1007/s12559-010-9063-2

Roberts, M. A., \& Besner, D. (2005). Stroop dilution revisited: Evidence for domain-specific, limited-capacity processing. Journal of Experimental Psychology: Human Perception and Performance, 31, 3-13. doi:https://doi.org/10.1037/0096-1523.31.1.3
Theeuwes, J., Kramer, A. F., \& Belopolsky, A. V. (2004). Attentional set interacts with perceptual load in visual search. Psychonomic Bulletin \& Review, 11, 697-702.doi:https://doi.org/10.3758/BF03196622

Torralbo, A., \& Beck, D. M. (2008). Perceptual-load-induced selection as a result of local competitive interactions in visual cortex. Psychological science, 19, 1045-1050. doi:https://doi.org/10.1111/ j.1467-9280.2008.02197.x

Treisman, A. (1960). Contextual cues in selective listening. Quarterly Journal of Experimental Psychology, 12, 242-248. doi:https://doi. org/10.1080/17470216008416732

Tsal, Y., \& Benoni, H. (2010). Diluting the burden of load: Perceptual load effects are simply dilution effects. Journal of Experimental Psychology: Human Perception and Performance, 36, 1645-1656. doi:https://doi.org/10.1037/a0018172

Wilson, D. E., Muroi, M., \& MacLeod, C. M. (2011).Dilution, not load, affects distractor processing. Journal of Experimental Psychology: Human Perception and Performance, 37, 319-335. doi:https://doi. org $/ 10.1037 / \mathrm{a} 0021433$ 